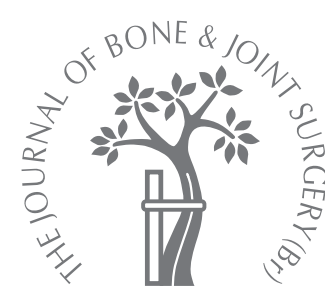

\title{
A comparison of augmentation techniques for reconstruction of the extensor mechanism following proximal tibial replacement in an experimental animal model
}

\author{
C. J. Pendegrass, \\ S. Sundar, \\ M. J. Oddy, \\ S. R. Cannon, \\ T. Briggs, \\ G. W. Blunn
}

From The Royal

National

Orthopaedic

Hospital, Stanmore, England

\begin{abstract}
We used an in vivo model to assess the use of an autogenous cancellous bone block and marrow graft for augmenting tendon reattachment to metallic implants. We hypothesised that augmentation of the tendon-implant interface with a bone block would enable retention of the graft on the implant surface, enhance biological integration, and result in more consistent functional outcomes compared with previously reported morcellised graft augmentation techniques.

A significant improvement in functional weight-bearing was observed between six and 12 weeks. The significant increase in ground reaction force through the operated limb between six and 12 weeks was greater than that reported previously with morcellised graft augmented reconstructions. Histological appearance and collagen fibre orientation with bone block augmentation more closely resembled that of an intact enthesis compared with the morcellised grafting technique. Bone block augmentation of tendon-implant interfaces results in more reliable functional and histological outcomes, with a return to pre-operative levels of weight-bearing by 24 weeks.
\end{abstract}

Failure to attach soft tissues to the massive endoprostheses used in tumour surgery and in complex revision arthroplasty can compromise functional outcome. Although numerous techniques have been employed to achieve softtissue reconstruction, ${ }^{1-8}$ no single method has consistently achieved superior results. Reconstruction of the extensor mechanism following replacement of the proximal tibia relies on successful reattachment of the patellar tendon to the implant to allow walking. We have previously described the use of a custom-made hydroxyapatite (HA)-coated titanium alloy implant for reattachment of the patellar tendon in an ovine model. ${ }^{9-11}$ We have shown that using morcellised autogenous bone and marrow graft results in good functional recovery when the tendon is transfixed to the implant using either a clamp lid ${ }^{9,10}$ or a Vicryl mesh construct. ${ }^{11}$ Although retention of the graft at the tendon-bone-HA interface was variable, resulting in only sporadic formation of bone and fibrocartilage at the developing tendon-implant neo-enthesis, the resulting structures were functional and histologically superior to non-augmented controls. However, considerable variations in both outcome measures were observed between individuals in the augmented groups. We believe that variation in the functional outcome depends largely on the biological interface that develops at the tendon-implant interface, and that retention of the graft at this site is critical for both biological regeneration and functional recovery.

In this study we used our previous model of in vivo tendon reattachment, ${ }^{9,10}$ with the addition of an autogenous cancellous bone block to the marrow graft to improve retention of the graft at the tendon-bone-HA interface. Our hypothesis was that augmentation of the tendon-implant interface with a bone block would improve graft retention, enhance biological integration and result in more consistent functional outcome compared with the augmentation using morcellised graft only. We assessed the consistency of function and morphology of the reconstructions of the extensor mechanism augmented by a bone block for up to 24 weeks. We also compared the functional and morphological data at six and 12 weeks from these reconstructions with data from animals that had received augmentation with autogenous morcellised bone graft to re-establish extensor mechanism function in a previous experiment. ${ }^{9,10}$

\section{Materials and Methods}

A titanium-alloy (Ti-6A1-4V) implant consisting of a base-plate with an H-shaped clamp lid with a series of $111 \mathrm{~mm}$ drill holes was used to 
simulate the surface of a proximal tibial replacement. The base-plate was coated with a $70 \mathrm{~mm}$ thick layer of plasmasprayed HA (Plasma Biotal, Tideswell, United Kingdom) and the tendon transfixed between the base-plate and the clamp lid with nine interlocking press-fit spikes, $1 \mathrm{~mm}$ in diameter and $6 \mathrm{~mm}$ long, to achieve mechanical fixation. The base-plate of the clamp isolated the recovering interface from the underlying bone, and the perforated lid enabled access from the overlying soft tissues.

The study was carried out under the UK Home Office Animals (Scientific Procedures) Act $1986 .^{12}$ We used 12 skeletally-mature Friesland ewes, weighing between $60 \mathrm{~kg}$ and $85 \mathrm{~kg}$. The tendon clamp was used to transfix the patellar tendon at an equivalent anatomical position on the anterior surface of the tibia. For the bone block group the tendon-implant interface was augmented with an autogenous bone block and a marrow graft. Three animals were killed after six weeks, five at 12 weeks and four at 24 weeks for histological analysis. Functional recovery was assessed using force-plate analysis of all surviving animals at six, 12, 18 and 24 weeks. The data at six and 12 weeks from these animals were compared with those of a previous group of six whose patellar tendon was attached using identical clamp devices and surgical procedures, with morcellised graft and marrow augmentation (autograft group). ${ }^{7,8}$

The animals were premedicated with an injection of xylazine hydrochloride $0.2 \mathrm{mg} / \mathrm{kg}$ (Bayer plc, Bury St Edmunds, United Kingdom). Anaesthesia was induced with intravenous midazolam (2.5 mg stat dose; Roche Products Ltd, Welwyn Garden City, United Kingdom) and ketamine hydrochloride $2 \mathrm{mg} / \mathrm{kg}$ (Fort Dodge Animal Health Ltd, Southampton, United Kingdom), and maintained with 3\% halothane (Merial Animal Health Ltd, Harlow, United Kingdom) and oxygen (4 1/min). A midline incision was made over the right stifle (knee) joint through which the patellar tendon was defined and elevated from the tibial tuberosity. An osteotomy of the tibial tubercle was undertaken to create a flat bone bed to which the base-plate was attached with two $2.7 \mathrm{~mm}$ self-tapping cortical bone screws (Synthes, Stratec Medical Ltd, Welwyn Garden City, United Kingdom). The clamp lid was assembled on to the baseplate to provide mechanical reattachment of the patellar tendon between the interlocking spikes. The tendon-HA interface was augmented with a fresh autogenous bone block $(10 \mathrm{~mm} \times 10 \mathrm{~mm} \times 2 \mathrm{~mm})$ and $1.5 \mathrm{ml}$ of bone marrow aspirate, which were harvested from the ipsilateral iliac crest and pressed on to the HA-coated base-plate prior to clamping the tendon into place. Subcutaneous doses of ceftiofur antibiotic $1 \mathrm{mg} / \mathrm{kg}$ (Pharmacia Upjohn, Stockholm, Sweden) were administered intra-operatively and for five post-operative days. Intramuscular buprenorphone hydrochloride (10 mg/kg; Reckitt \& Colman Products Ltd, Melton Mowbray, United Kingdom) was given during the procedure and for one day afterwards. The animals were permitted free mobilisation in a pen $(3 \mathrm{~m} \times 4 \mathrm{~m})$ immediately after operation, and were group housed after a period of two weeks.
Force-plate analysis. The animals underwent force-plate analysis (Kistler Biomechanics Ltd, Alton, United Kingdom) pre-operatively, and at six, 12, 18 and 24 weeks after operation. Data for 12 unimpeded walks over the forceplate were collected from both hind limbs of each animal. Using custom-written software, the mean peak vertical ( $z$ co-ordinate) component of the ground reaction force, normalised for the weight of the animal $\left(\mathrm{F}_{\max } /\right.$ weight $)$, was determined for both hind limbs. The mean peak vertical $\left(\mathrm{F}_{2}\right)$ ground reaction force of the right hind limb was calculated as a percentage of the left (operated/non-operated as a percentage).

Histological analysis. The clamped specimens were harvested en bloc by dissection through the mid-substance of the patellar tendon and the proximal tibia below the distal margin of the base-plate. The samples were fixed in $10 \%$ formalin, dehydrated in an ascending alcohol series, defatted in chloroform for five days, and embedded in LR White Hard Grade Resin (London Resin Company Ltd, Reading, United Kingdom). Sections were cut, ground and polished to $100 \mathrm{~mm}$, stained with toluidine blue and paragon, and analysed using a Zeiss microscope with Axioimage (version 4.4) image analysis software (Carl Zeiss Ltd, Welwyn Garden City, United Kingdom). Sections were taken at onethird width intervals across each tendon-implant specimen. For each section, five fields of view were analysed along the length of the base-plate at a magnification of $\times 100$. Double-blind quantitative analysis of the orientation of the collagen fibres at the tendon-bone interface was performed using Adobe Photoshop CS2 (Adobe Systems Inc., San Jose, California) by measuring the angles of orientation of the collagen fibres using the base-plate as a point of reference. The data were compared with those from the autograft group, and from six intact patellar tendon-bone entheses which were similarly measured, using the tangent to the surface of the tibial tuberosity as a point of zero reference. Semi-quantitative analysis of the bone graft-tendon interface was carried out. The interface tissues were described on the basis of their histological appearance as either perforating fibrous, where collagen fibres were observed inserting directly into bone without transitional regions of cartilage, or fibrocartilaginous, when the tendon-bone insertions progressed through transitional regions of fibrocartilage and mineralised fibrocartilage with clear evidence of chondrocyte-like cells in the lacunae, and were expressed as the interface percentage at six and 12 weeks.

Statistical analysis. The numerical data were analysed using non-parametric tests with SPSS for Windows version 12.0 (SPSS, Chicago, Illinois). Wilcoxon's signed-ranks test was used to assess differences in functional weight-bearing in the bone block group to account for the variability between individuals within the group. The differences in functional weight-bearing between the bone block and autograft groups, and in $\mathrm{F}_{\max } /$ weight for the operated and control limbs between six and 12 weeks, were determined using the Mann-Whitney U and Wilcoxon's signed-ranks 

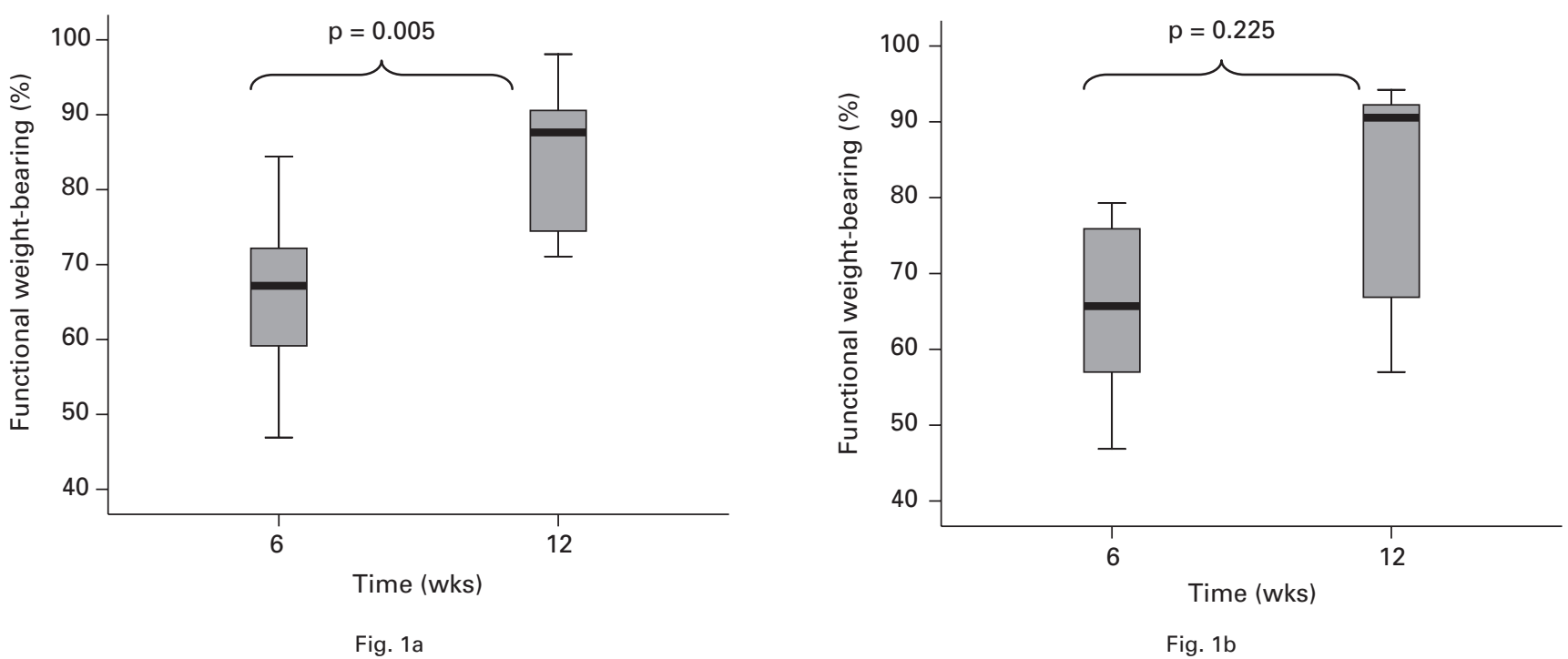

Box and whisker plots showing the change in functional weight-bearing (\%) for animals a) with reconstruction of the extensor mechanism augmented by bone block and b) with reconstruction of the extensor mechanism augmented by morcellised bone grafts.

tests, respectively. Data are presented as median values with $95 \%$ confidence intervals $(\mathrm{CI})$, and results were considered significant at $\mathrm{p}=0.05$.

\section{Results}

Force-plate analysis. All the animals in the bone block group were fully mobile within 24 hours of surgery and there were no complications in the peri- or post-operative periods. Figure $1 \mathrm{a}$ shows the operated/control limb percentage for the bone block group over 12 weeks. Figure $1 \mathrm{~b}$ shows the data from the autograft group ${ }^{9,10}$ over 12 weeks for comparison. The variability between individuals within a group is demonstrated by the confidence intervals shown in the box plots.

At six and 12 weeks, the median ground reaction force of the operated limb $\left(\mathrm{F}_{\mathrm{z}}\right)$, expressed as a percentage of the unoperated leg was $67.17 \%$ (95\% CI 59.32 to 73.02$)$ and $87.64 \%$ (95\% CI 78.09 to 91.69) for the bone block group. A significant improvement was observed between the two time points ( $\mathrm{p}=0.005$ ) (Fig. 1a). The equivalent data for the autograft group were $65.70 \%$ (95\% CI 58.79 to 72.24 ) and $90.55 \%$ (95\% CI 58.99 to 101.44$),{ }^{9,10}$ with no significant improvement owing to the large confidence interval observed at 12 weeks ( $\mathrm{p}=0.225)$ (Fig. 1b). When comparing the data between the animals in the current study, who received bone block augmentation, with those with augmentation with a morcellised graft, no significant differences were observed at either six $(p=0.817)$ or 12 $(\mathrm{p}=0.806)$ weeks. At 18 and 24 weeks, the median ground reaction force of the operated $\operatorname{limb}\left(\mathrm{F}_{\mathrm{z}}\right)$, expressed as a percentage of the un-operated leg, was $87.90 \%$ (95\% CI 82.91 to 97.35 ) and $97.47 \%$ (95\% CI 94.36 to 99.82 ) for the bone block group. No significant differences were observed between 12 and 18 weeks ( $p=0.310$ ) or between 18 and 24 weeks $(\mathrm{p}=0.176)$.

In the bone block group the change in $\mathrm{F}_{\max } /$ weight between six and 12 weeks for the operated limb was $8.3 \mathrm{Nkg}^{-1}$. By comparison, the equivalent change observed in the group with augmentation with morcellised graft was 4.5 $\mathrm{Nkg}^{-1}$. Augmentation with a bone block resulted in a significant increase in $\mathrm{F}_{\max } /$ weight between six and 12 weeks for the operated limb compared with the morcellised grafting group $(\mathrm{p}=0.049)$ (Fig. 2). There were no significant differences in the change in $\mathrm{F}_{\max }$ /weight for the opposite limb between the groups at the same time points $(\mathrm{p}=0.600)$, indicating that the animals did not compensate for a reduction in function in operated limb function with their contralateral limbs.

Histological analysis. The insertion of the patellar tendon from the six intact specimens consisted of normal tendon attached to the underlying bone via a direct type enthesis, with a median orientation of the collagen fibres tangential to the insertion of $33.00^{\circ}\left(29.58^{\circ}\right.$ to $\left.40.24^{\circ}\right)$. The median orientation in the bone block group was $31.32^{\circ}\left(29.62^{\circ}\right.$ to $\left.34.89^{\circ}\right)$, which was not significantly different from the intact controls $(\mathrm{p}=0.409)$. However, the median orientation previously seen with augmentation with morcellised graft in the autograft group was $19.80^{\circ}\left(17.69^{\circ}\right.$ to $\left.21.77^{\circ}\right)$, which was significantly lower than in the intact controls $(\mathrm{p}=0.000)$, and the current bone block group specimens $(\mathrm{p}=0.000)$.

Table I summarises the tissue present at the bone grafttendon interface. At six and 12 weeks, the interfaces in the bone block group were significantly more fibrocartilaginous than those of the autograft group ( $\mathrm{p}=0.004$ and 0.002 , respectively). 


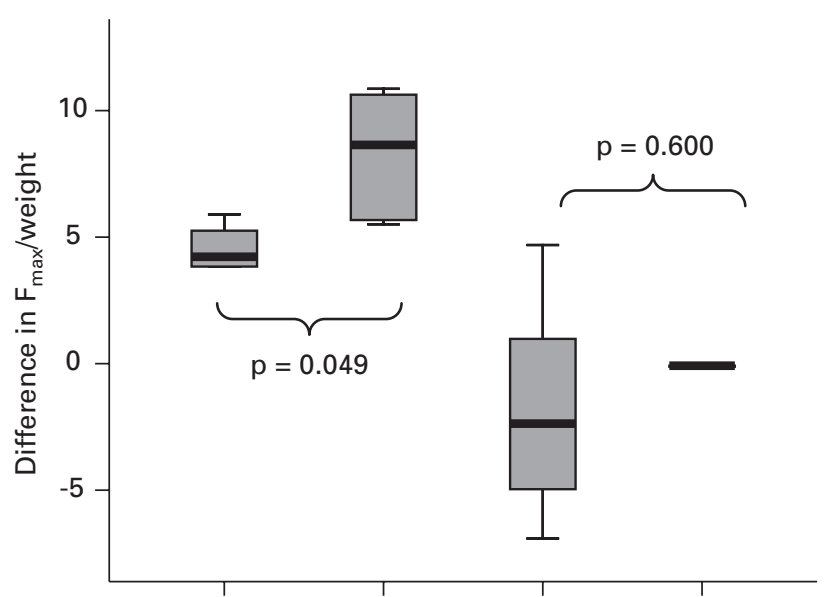

Autograft Bone block Autograft Bone block

Operated

Control

Fig. 2

Box and whisker plot showing the difference in $\mathrm{F}_{\max } /$ weight between six and 12 weeks for the operated and control limbs of animals with bone block and morcellised bone graft (autograft) augmentation of reconstructions of the extensor mechanism.

At six weeks in the bone block group, the tendon running from the patella towards the clamp consisted of dense crimped collagenous tissue with parallel orientated collagen fibres interspersed with elongated fibroblast nuclei. This morphology extended into the clamped region of the mid-substance of the tendon, where the collagen fibres were aligned along the line of tensile loading. However, areas of disorganisation were observed. The nuclei of the fibroblast were oval, and a mixed inflammatory infiltrate was seen. These observations were consistent with those of the autograft group at six weeks. Despite this, the boneimplant interface was markedly different in the two groups. In the autograft group at six weeks, bone was undergoing active remodelling with osteoblasts and multinucleated osteoclasts clearly visible. Regions of the tendon-bone-HA interface were interrupted with a layer of disorganised fibrous tissue with no bone formation. Where bone was seen attached to the HA coating, the interface between the bone and tendon consisted of perforating collagen fibres, but without transitional fibrocartilage. However, in the bone block group, bone was continuous with the HA coating (Fig. 3a), and there was a clear tendon-bone interface. The interface between the bone and the tendon consisted predominantly of perforating collagen fibres extending from the tendon and running on to the underlying bone surface, with regions of transitional fibrocartilage (Fig. 3b). The residual bone within the clamp appeared to be undergoing active remodelling, with multinucleated osteoclasts in Howship lacunae resorbing bony trabeculae, and columnar osteoblasts with thick osteoid seams depositing new bone.
By 12 weeks in the bone block group, the mid-substance of the tendon external to and within the clamp consisted of dense crimped collagenous tissue orientated in the same direction. The nuclei of the fibroblasts were elongated throughout, and there was no evidence of infiltration with inflammatory cells. These findings were consistent in both grafting techniques, but in the autograft group there was an incomplete bone-HA interface at 12 weeks. The tendonbone interface had developed a layered structure with clearly defined regions of fibrocartilage. However, bone islands were found away from the HA layer. Where bone graft was not retained on the HA-coated base-plate, the interface consisted of a dense collagenous tissue orientated parallel to the surface of the implant (Fig. 4a). In the bone block group the interface was more organised, with the tendon-bone-HA interface extending over the entire length of the base-plate (Fig. 4b). At 12 weeks the tendon-bone interface was composed of regions of fibrocartilage and mineralised fibrocartilage (Fig. 4c). The bone block had undergone significant remodelling and new bone filled the clamped space. These appearances were still present at 24 weeks, demonstrating the characteristics of a direct enthesis.

\section{Discussion}

We investigated the use of an autogenous bone block and marrow augmentation for reconstruction of the extensor mechanism in the knee. We also compared the findings with our previous studies of augmentation with a morcellised graft and bone marrow. ${ }^{9,10}$ The aims were threefold. First, to determine whether augmentation with an autogenous bone block would result in improved graft retention and greater functional recovery than with morcellised bone graft; secondly, to determine which of these two grafts led to the development of a more mature enthesis; and finally to investigate extensor function and the histological appearance of the interfaces augmented by a bone block beyond 12 weeks.

In the autograft group retention of the morcellised graft varied considerably between individuals, and the tendonbone-HA interface occupied a maximum of $66 \%$ of the length of the base-plate. ${ }^{9}$ With augmentation with a bone block, retention of the graft was more consistent and the tendon-bone-HA interface occupied the whole surface of the base-plate. In the bone block specimens the tendonbone interface developed to resemble that of a normal direct enthesis by 12 weeks, and appeared unchanged at 24 weeks. With morcellised graft augmentation, limited retention of the graft on the HA-coated base-plate meant that the tendon-implant interface was not morphologically consistent. Where bone graft was retained a normal direct enthesis was observed, but these regions alternated with zones of fibrous tissue encapsulation where the graft was no longer present at the tendon-implant interface. This compromised the development of the enthesis and indicated that bone formation is necessary for morphological and functional recovery of the enthesis. 
Table I. A summary of tissue types present at the bone graft-tendon interface in the bone block and autograft groups

\begin{tabular}{lcll}
\hline Group & Post-operative duration (wks) & $\begin{array}{l}\text { Median perforating fibrous (\%) } \\
\text { (95\% Cl) }^{*}\end{array}$ & $\begin{array}{l}\text { Median fibrocartilagenous (\%) } \\
\text { (95\% Cl) }\end{array}$ \\
\hline Bone block & 6 & $65.90(65.15$ to 67.09) & 34.35 (33.65 to 35.22) \\
& 12 & $11.10(9.89$ to 12.21$)$ & $89.15(88.40$ to 90.51$)$ \\
Autograft & 6 & $84.05(80.02$ to 88.08$)$ & $16.10(14.74$ to 17.99$)$ \\
& 12 & $24.45(23.04$ to 26.99$)$ & 76.50 (75.34 to 77.84) \\
\hline
\end{tabular}

*95\% Cl, 95\% confidence interval

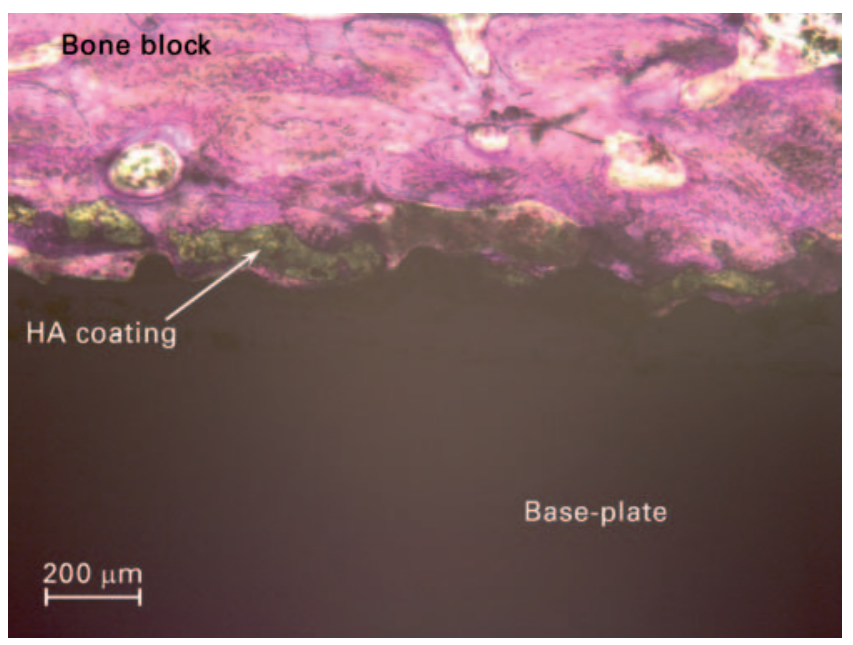

Fig. 3a

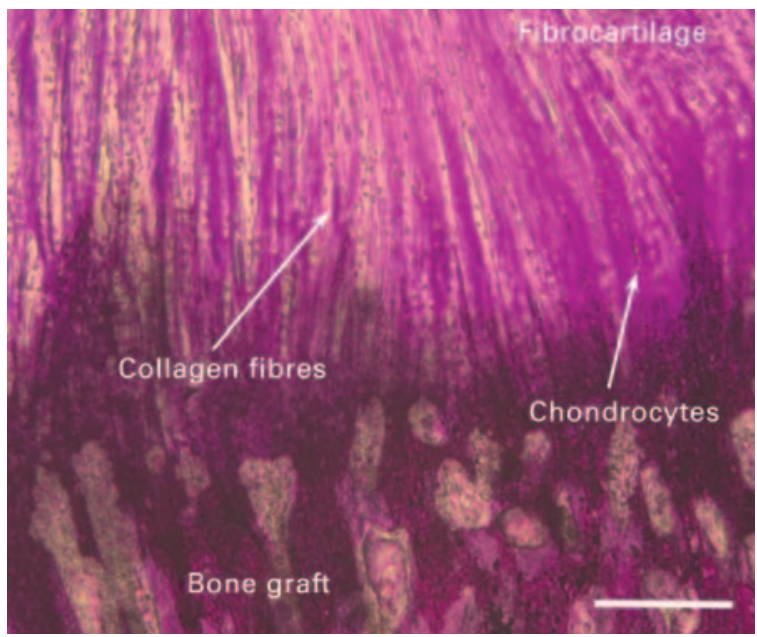

Fig. 3b

a) The bone-hydroxyapatite (HA)-implant interface at six weeks in a reconstruction augmented by a bone block showing bone continuous with the HA coating. Specimen stained with toluidine blue and paragon. Bar $=200 \mu \mathrm{m}$. b) Perforating collagen fibres extending from the tendon into bone in a reconstruction augmented by a bone block at six weeks. Specimen stained with toluidine blue and paragon. Bar $=50 \mu \mathrm{m}$.

Large differences in functional recovery, as indicated by large confidence intervals, were observed between individuals receiving augmentation with morcellised graft. This correlates with the limited graft retention at the implant surface and the resulting variable fibrocartilaginous interface. Good graft retention was observed in all animals receiving a bone block, and this correlated with the consistency in functional recovery and the narrow confidence intervals for the animals in the bone block group. Forceplate analysis has been used to assess limb function both clinically and experimentally, ${ }^{13-15}$ and forces through the patellar tendon have been shown to be directly related to the vertical ground reaction force $\left(\mathrm{F}_{\mathrm{z}}\right)$ in vivo. ${ }^{16}$ Our model shows that graft retention and the structure of the developing enthesis are mirrored by the functional recovery of the interface. Despite the lack of significant differences in functional weight-bearing between the groups at each time point, a significant improvement was observed for the bone block group between six and 12 weeks, indicating a faster functional recovery than in the morcellised graft group. There was a large variation in functional recovery in the morcellised augmented group at six and 12 weeks, with no significant improvement between these time intervals. The spread of the data shows that although some individuals recover well between six and 12 weeks, there are a number who do not. Consequently, for clinical application, augmentation of tendon-implant interfaces with morcellised graft could be associated with large variations in functional recovery between patients. In the bone block group the confidence interval at 12 weeks was narrower, indicating that functional recovery was more consistent between individuals. This suggests that this procedure could produce more reliable functional outcomes, with a reduction in variability between patients following proximal tibial replacement.

Various biological materials have been used as graft tissue in the musculoskeletal system. Higuera et $\mathrm{al}^{17}$ described the use of an allogenic bone plate augmented with either autogenous bone and marrow graft or recombinant human osteogenic protein-1 (rhOP-1) for reattachment of a tendon to a metallic implant in a canine model. They considered that direct tendon-bone attachment could be achieved using both augmentation regimens, and that 


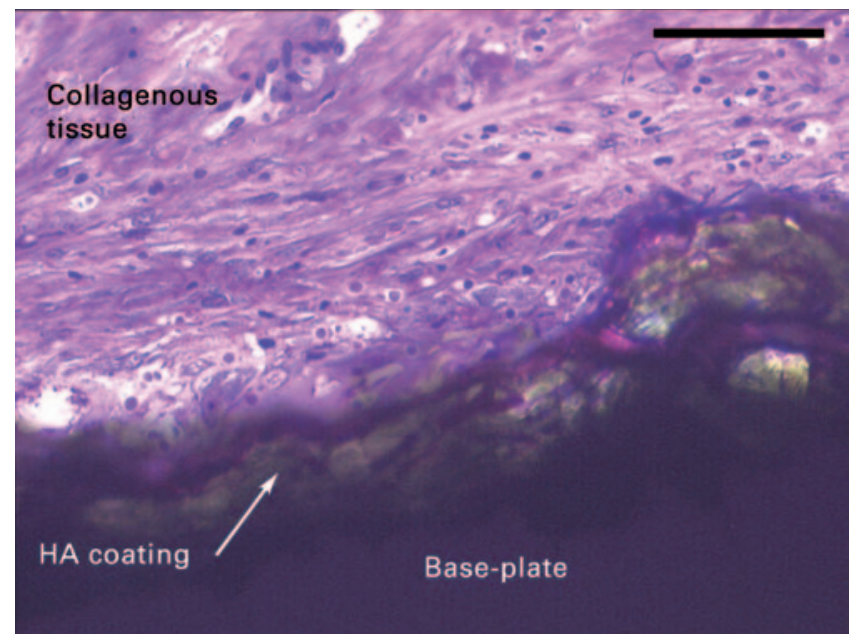

Fig. 4a

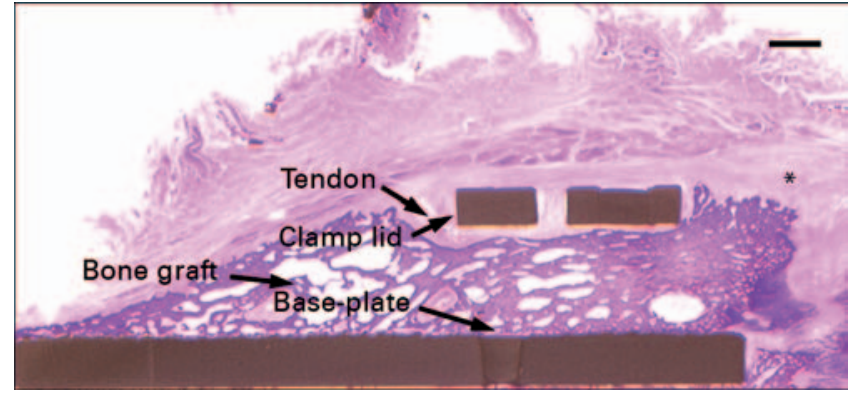

Fig. 4b

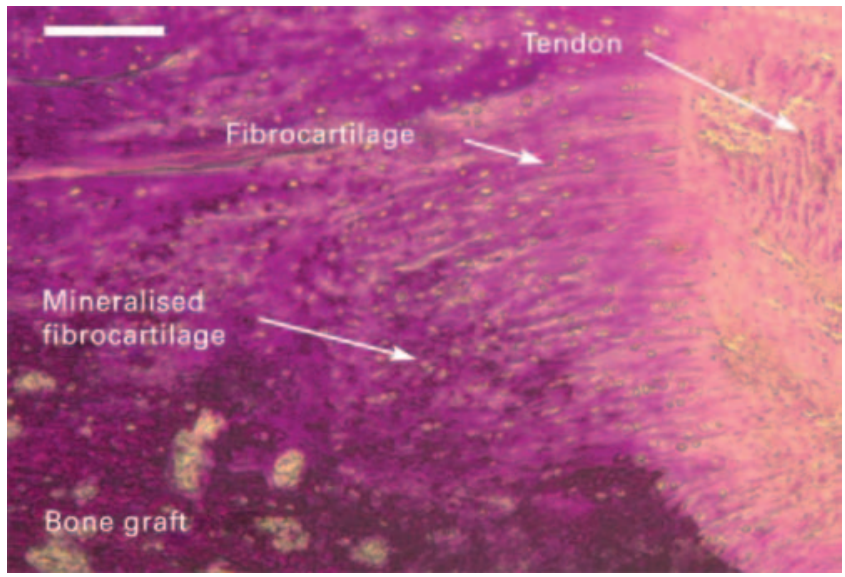

Fig. 4c

a) A region where bone graft was not retained at the hydroxyapatite (HA) surface of the clamp in a reconstruction augmented by morcellised bone graft at 12 weeks, showing encapsulation of the base plate. Specimen stained with toluidine blue and paragon. Bar = 100 um. b) A low-power image of a reconstruction augmented by a bone block showing bone graft in contact with the whole HA-coated base-plate at 12 weeks. Specimen stained with toluidine blue and paragon. Bar $=2 \mathrm{~mm}$. c) A higher-magnification image of the region marked* in Fig. $4 \mathrm{~b}$ showing the layered enthesis observed in reconstructions augmented by bone block at 12 weeks. Specimen stained with toluidine blue and paragon. Bar $=100 \mu \mathrm{m}$.

autogenous bone and marrow graft could be successfully substituted with rhOP-1. However, no significant differences in recovery of weight-bearing, ultimate tensile strength or histological appearance were observed, irrespective of the method employed. Clinically, allograft is often used to overcome the limitations associated with autograft, which include lack of supply, the necessity for a second operation site, and increased morbidity from the donor site. ${ }^{18}$ However, allograft has been shown to have reduced osteoinductive and osteoconductive properties, and to induce an immune response. ${ }^{19,20}$ Although allograft bone marrow cells have been implicated as a major source of immunogenicity, ${ }^{21}$ removing them does not prevent acti- vation of $\mathrm{T}_{\text {cells }}{ }^{22}$ which renders the use of acellular allograft equally challenging to the immune system of the recipient. Autograft does not induce a foreign-body immune response and is therefore accepted and integrated into the recipient without the limitations associated with allogenic graft material. Although the majority of cells transplanted into autogenous cancellous bone die, mesenchymal stem cells have been shown to survive and proliferate under certain conditions. ${ }^{20}$ Autogenous cancellous bone grafts are rapidly revascularised and invaded by granulation tissue, undergoing bone resorption and new bone formation after only a few weeks. ${ }^{23,24}$ They tend to repair completely with time in a favourable mechanical environ- 
ment, but incorporation is generally influenced by the size of the graft and the species of the host. ${ }^{22}$ We feel that the clamp environment is conducive to incorporation of autogenous cancellous bone graft and remodelling, which could be indicative of a favourable clinical outcome.

The need for the second operation site and donor site morbidity did not contribute adversely to our findings. In both experimental groups, a second operation site was used and functional weight-bearing was not significantly impaired. Based on our functional and histological findings, and the considerable limitations associated with the use of allograft, ${ }^{19-22}$ we advocate the use of autogenous bone graft for augmentation of tendon reconstructions to metallic implants.

We have shown that using a bone block to augment the healing tendon-implant interface results in superior graft retention compared with the use of morcellised graft. The structure of the tendon-bone-implant interface was predominantly of the direct type at 24 weeks. This had developed consistently across the length of the insertion site and correlated with successful functional recovery. More reliable functional outcomes were observed with the use of bone block augmentation, and normal levels of functional weight-bearing were regained more quickly.

No benefits in any form have been received or will be received from a commercial party related directly or indirectly to the subject of this article.

\section{References}

1. Malawer MM, McHale KA. Limb-sparing surgery for high-grade malignant tumours of the proximal tibia: surgical technique and a method of extensor mechanism reconstruction. Clin Orthop 1989;239:231-48.

2. Eckardt JJ, Matthews JG 2nd, Eilber FR. Endoprosthetic reconstruction after bone tumor resections of the proximal tibia. Orthop Clin North Am 1991;22:149-60.

3. Jeon DG, Kawai A, Boland P, Healey JH. Algorithm for the surgical treatment of malignant lesions of the proximal tibia. Clin Orthop 1999;358:15-26.

4. Ozaki T, Kunisada T, Kawai A, Takahara Y, Inoue H. Insertion of the patella tendon after prosthetic replacement of the proximal tibia. Acta Orthop Scand 1999;70:527-9.

5. Bickels J, Wittig JC, Kollender Y. Reconstruction of the extensor mechanism after proximal tibia endoprosthetic replacement. J Arthroplasty 2001;16:856-62.

6. Gosheger G, Hillmann A, Lindner N, et al. Soft tissue reconstruction of megaprostheses using a trevira tube. Clin Orthop 2001;393:264-71.
7. Reach JS, Dickey ID, Zobitz ME, et al. Direct tendon attachment and healing to porous tantalum: an experimental animal study. J Bone Joint Surg [Am] 2007;89A:1000-9.

8. Itälä A, Heijink A, Leerapun $T$, et al. Successful canine patellar tendon reattachment to porous tantalum. Clin Orthop 2007;463:202-7.

9. Pendegrass CJ, Oddy MJ, Cannon SR, et al. A histomorphological study of tendon reconstruction to a hydroxyapatite-coated implant: regeneration of a neo-enthesis in vivo. J Orthop Res 2004;22:1316-24.

10. Oddy MJ, Pendegrass CJ, Goodship AE, et al. Extensor mechanism reconstruction after proximal tibial replacement. J Bone Joint Surg [Br] 2005;87-B:873-8.

11. Pendegrass CJ, Oddy MJ, Sundar S, et al. The novel use of resorbable Vicryl mesh for in vivo tendon reconstruction to a metal prosthesis. J Bone Joint Surg [Br] 2006;88-B:1245-51.

12. No authors listed. Animals (Scientific Procedures) Act 1986. http:// www.archive.official-documents.co.uk/document/hoc/321/321-xa.htm (date last accessed 21 February 2008).

13. Otis JC, Lane JM, Kroll MA. Energy cost during gait in osteosarcoma patients after resection and knee replacement and after above-the-knee amputation. J Bone Joint Surg [Am] 1985;67-A:606-11.

14. Ghosh P, Read R, Armstrong S, et al. The effects of intraarticular administration of hyaluronan in a model of early osteoarthritis in sheep. 1: gait analysis and radiological and morphological studies. Semin Arthritis Rheum 1993;22:18-30.

15. Kotani Y, Tokuhiro A. Kinesiological study of the push-up motion in spinal injury patients; involving measurement of hand pressure applied to a force plate. Acta Med Okayama 2002; 56:75-82

16. Korvick DL, Cummings JF, Grood ES, et al. The use of an implantable force transducer to measure patellar tendon forces in goats. J Biomech 1996;29:557-61.

17. Higuera CA, Inoue $\mathbf{N}$, Lim JS, et al. Tendon reattachment to a metallic implant using an allogenic bone plate augmented with rhOP-1 vs autogenous cancellous bone and marrow in a canine model. J Orthop Res 2005;23:1091-9.

18. Perry CR. Bone repair techniques, bone graft and bone graft substitutes. Clin Orthop 1999;360:71-86.

19. Goldberg VM, Akhavan S. Biology of bone grafts. In: Friedlaender GE, Mankin HJ, Goldberg NM, eds. Bone grafts and bone graft substitutes. Rosemont: American Academy of Orthopaedic Surgeons, 2006:1-8.

20. Bauer TW, Muschler GF. Bone graft materials: an overview of the basic science. Clin Orthop 2000;371:10-27.

21. Esses SI, Halloran PF. Donor marrow-derived cells as immunogens and targets for the immune response to bone and skin allografts. Transplantation 1983;35:169-74.

22. Horowitz MC, Friedlaender GE. Induction of specific T-cell responsiveness to allogenic bone. J Bone Joint Surg [Am] 1991;73-A:1157-68.

23. Miclau T, Lindsey RW, Probe R, Rahn BA, Perren SM. Autogenous cancellous bone graft incorporation in a gap defect in the canine femur. J Orthop Trauma 1996;10:108-13.

24. Burchardt H. The biology of bone graft repair. Clin Orthop 1983;174:28-42. 